\title{
Análisis de las ventajas y desventajas del Big Data y el Cloud Computing en el proceso de la toma de decisiones de lasempresas que practican comercio electrónico
}

\section{Analysis of the advantages and disadvantages of Big Data and Cloud Computing in the decision-making process of companies that practice electronic commerce}

\author{
Ing. Ricardo Rafael Coello Yagual, $\mathrm{MSc}^{1}$ \\ ricardo_mvc@hotmail.com \\ Econ. Johnny Daniel Parrales Ponce 2 \\ daniel1parra@hotmail.com
}

Reciido: 1/9/2019; Aceptado: 1/11/2019

\begin{abstract}
RESUMEN
El presente trabajo de investigación refleja el análisis de las ventajas y desventajas del Big Data y el Cloud Computing, en el proceso de toma de decisiones de las empresas que practican comercio electrónico en el Ecuador. Para realizar el análisis, se identificó las variables esenciales que influyen en el proceso de toma de decisiones. Y se presenta la propuesta del Big Data y el Cloud Computing como herramientas de interpretación para la información no estructurada que proviene de diferentes medios.
\end{abstract}

Palabras Clave: medios sociales, comercio electrónico, Big Data, cloud computing, Inteligencia de Negocios

\section{ABSTRACT}

This research paper reflects the analysis of the advantages and disadvantages of Big Data and Cloud Computing, in the decision-making process of companies that practice electronic commerce in Ecuador. To perform the analysis, the essential variables that influence the decision- making process were identified. And the Big Data and Cloud Computing proposal is presented as interpretation tools for unstructured information that comes from different media.

Keywords: social media, electronic commerce, computing, business, intelligence

Big Data,

cloud

\footnotetext{
1 Universidad Tecnológica Empresarial de Guayaquil, Guayas, Ecuador.

2 Universidad Tecnológica Empresarial de Guayaquil, Guayas, Ecuador.
} 


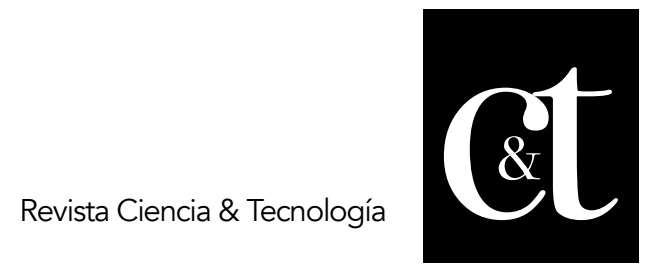

No. 25, 31 de enero de 2020

ISSN impreso: 1390 - 6321

ISSN online: 2661 - 6734

\section{Introducción}

En la actualidad las empresas se encuentran en una búsqueda de ventajas competitivas que las lleve a perdurar en el tiempo, este fenómeno se fundamenta en buscar continuamente satisfacer las necesidades del cliente, es decir que el proceso de toma de decisiones tiene una gran importancia en la creación valor para la organización.

La empresa actualmente busca mayores posibilidades de éxito y mantenerse por delante de su competencia, es decir la influencia de la globalización ha generado consumidores cada día más exigentes, así también "depurar" los requerimientos que solicitan los clientes se convierte para la empresa en una prioridad de éxito en la consecución de los resultados que espera (Martínez \& Lara, 2014). Las organizaciones necesitan saber sobre el entorno que las rodea, por ello se vuelve muy importante la buena interpretación de la información que proviene de las diferentes fuentes que se manejan actualmente; ante ello las tecnologías de la información y la comunicación se han convertido en las herramientas que las empresas utilizan no solo para adquirir tecnología, sino que actualmente son estrategias competitivas que intervienen en el proceso de la toma de decisiones de las empresas. A través del proceso de investigación se encontró los siguientes requerimientos:

estabilidad macroeconómica, apertura y acceso a mercados internacionales o la complejidad de la regulación para el sector empresarial, la infraestructura regional y la competitividad de las empresas (Bonales \& Zamora, 2015).

En el presente estudio a través de la investigación bibliográfica se hace referencia a una parte importante del tercer nivel, es decir a las herramientas que se pueden utilizar para analizar la información que necesita la empresa, en este caso la herramienta Big Data. También se analizará la propuesta de la implementación de Cloud Computing, como alternativa para el almacenamiento de datos, evidenciando que las empresas a través de la implementación del comercio electrónico generan gran volumen de información que debe ser analizada por las herramientas como el Big Data, así también almacenados de una manera estratégica y de bajo costo para la organización, como lo es el Cloud Computing.

Siguiendo nuestra investigación se podría indicar que el volumen de datos generados por diferentes fuentes de información crece exponencialmente, sólo en los últimos dos años la cifra mundial ha aumentado un 92\% (Tascán, 2013).

\section{Desarrollo}

La sociedad se encuentra en la era del Big Data, que se manifiesta por la generación de grandes cantidades de datos estructurados y no estructurados en tiempo real o diferido, los cuales necesitan ser analizados por las empresas para crear valor, esta era se caracteriza por lo dinámico de la creación de la información, es decir que "no sólo por el tamaño del conjunto de datos que se encuentran disponibles, sino que también posee otros atributos" (Martínez \& Lara, 2014); así también los autores identifican tres elementos que caracterizan a este fenómeno, llamados como: el volumen, la velocidad y la variedad. Algunos autores añaden un cuarto valor que es conocido como la veracidad. Es importante comprender que además de los datos estructurados, que son aquellos que provienen de fuentes de información conocidas, considerados también fáciles de medir y analizar por medio de los sistemas tradicionales, existen otros en la actualidad conocidos como los datos no estructurados, que son aquellos que provienen de la web, de las cámaras de los 


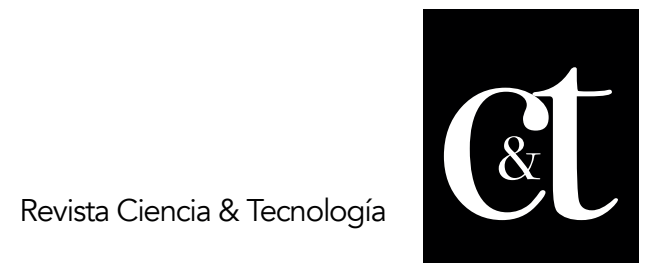

No. 25, 31 de enero de 2020

ISSN impreso: 1390 - 6321

ISSN online: 2661 - 6734

móviles y videos, de las redes sociales, de los sensores de las ciudades y edificios.

Las empresas en la actualidad presentan una gran dificultad para analizar estos datos no estructurados ya sea por la rapidez con la que se generan o por el volumen de contenido que poseen (Tascán, 2013). En el universo empresarial y el mundo de los negocios, se ha utiliza mucho el término Business Intelligence (BI) que no es otra cosa que el conjunto de estrategias y herramientas que una empresa tiene a su disposición para poder analizar los datos de su organización (Cueva, 2014), actualmente con las herramientas BI se realizan predicciones y análisis.

Big Data se lo relaciona con la minería de datos, que "es un campo de las Ciencias de la Computación que intenta descubrir los patrones en grandes volúmenes de datos" (Tascán, 2013), la minería de datos que es parte del BI, utiliza elementos de inteligencia artificial y estadística para la depuración de la información, este aspecto lo relaciona al Big Data que utiliza también este procedimiento para crear valor de la información. Así como las tecnologías mejoran exponencialmente, así también las herramientas habituales de trabajo cambian, es así que el talento humano de las organizaciones pasó de la "hoja de Excel a Hadoop, un software que permite trabajar miles de nodos distribuidos y con peta bytes de información" (Tascán, 2013).

\section{El Cloud Computing - una solución para la gestión en los negocios}

La tecnología evoluciona de manera acelerada haciendo que los programas que posee la empresa queden obsoletos rápidamente, en ocasiones la inversión que realizó la compañía no alcanzó a recuperarla, ya que "es una de las primeras que debe realizar $y$, en muchas ocasiones una de las que más parte del presupuesto requiere" (Abelar \& Hernández, 2010).

En la actualidad el modelo de negocio de Cloud Computing es una ventaja competitiva para la organización que busca "racionalizar el gasto en tecnología de la información permitiendo concentrar los recursos financieros y de personal en la actividad principal del negocio" (Abelar \& Hernández, 2010), siendo este un factor que permite la creación de valor y sostenimiento de la organización, al no tener como prioridad disponer de mucho dinero en el montaje de la empresa, proporcionándoles una infraestructura robusta y confiable para la gestión de manejo de datos, pues sólo se necesitan computadores de características normales y conexión a internet para el uso de los programas que necesiten (Mamani, 2014).

Cloud Computing es una estrategia de bajo costo y facilidad para la organización, que se ha convertido en una buena alternativa que apoya el emprendimiento al permitirle a los miembros de la empresa que "solo se concentren en el objeto del negocio, sin que los sistemas de información se conviertan en un problema de tiempo, conocimiento o dinero" (Abelar \& Hernández, 2010).

Para implementar Cloud Computing en la empresa es importante conocer el negocio, es decir saber que existen procesos, servicios requeridos y ofrecidos, flujos de información y quienes intervienen en ellos, lo cual permitirá obtener la mejor decisión en la búsqueda de optimizar la gestión de la información, "esto también nos brindará un análisis mayor en lo que se necesita en términos de infraestructura, plataforma y software" (Mamani, 2014).

Es importante indicar que el Cloud Computing es una metodología de trabajo ya que por medio de "la combinación de varias tecnologías de la información ya existentes y diferentes procedimientos, permite una nueva forma de hacer el trabajo dentro de una 


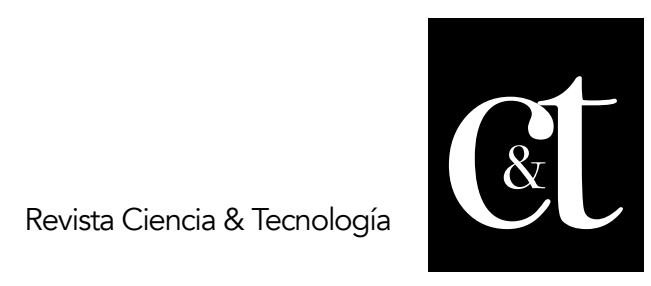

No. 25, 31 de enero de 2020

ISSN impreso: 1390 - 6321

ISSN online: 2661 - 6734

organización" (Abelar \& Hernández, 2010).

\section{Planteamiento y formulación del problema}

A través del planteamiento y formulación del problema se evidencia la existencia de ventajas y desventajas del Big Data y el Cloud Computing en el proceso de la toma de decisiones de las empresas. Entre los síntomas más importantes que se encontraron tenemos:

- Los datos no estructurados son elementos de información y se han convertido ventaja competitiva para la organización, así también para las pymes se han constituido en el motor de desarrollo comercial (El Comercio, 2015), es decir la existencia de herramientas que ayuden en el proceso de minería de datos se ha convertido en una urgente necesidad actualmente.

- La web, los medios sociales y los datos estructurados se han convertido junto con las herramientas de inteligencia de negocios en índices de competitividad en el Ecuador. (El Universo, 2016), por ello es necesario el análisis de las ventajas y desventajas que genera el uso de esta tecnología en las organizaciones.

Siguiendo la línea de investigación se encontró las causas que afectan a los síntomas anteriormente expuestos, entre ellas tenemos:

- Actualmente la empresa emergente digital recibe la información que proviene de la web semántica y los medios convencionales de información (datos estructurados), pero dentro del mundo del mundo de los negocios, el conocer las necesidades de los clientes, responder a sus inquietudes se ha vuelto un indicador de competitividad "y la venta de sus productos y servicios permite el crecimiento económico de las mismas". (Crespo, 2013).

- El Big Data y el Cloud Computing se convierten en una ventaja para los negocios que buscan comprender y analizar continuamente la interacción con sus clientes, es decir la creación de nuevas oportunidades de negocios (Aguirre \& Andrade, 2006), lo cual ha permitido el incremento en sus ingresos, lo cual se constituye en una información valiosa para el objeto de este estudio.

Utilizando el formato de investigación se presentan las siguientes preguntas de pronóstico:

- ¿Cuál debería ser el papel que realice el "científico de datos", como la persona encargada de la interpretación de la información que proviene de las fuentes de información no estructuradas, a través de la implementación de herramientas de Big Data?

- ¿Qué ventajas y desventajas tendría en los recursos disponibles de las empresas la implementación de Cloud Computing (almacenamiento en la nube)?

Se encontró entonces, el siguiente control del pronóstico:

- Actualmente las empresas interpretan la información no estructurada que proviene de los medios sociales, video y audio a través de los Sistemas de Información Gerencial, pero se hace necesario la utilización del Big Data por el incremento en volumen de información y rapidez, siendo la inteligencia artificial hoy en día un aliado estratégico para la organización en el proceso de la toma de decisiones. 


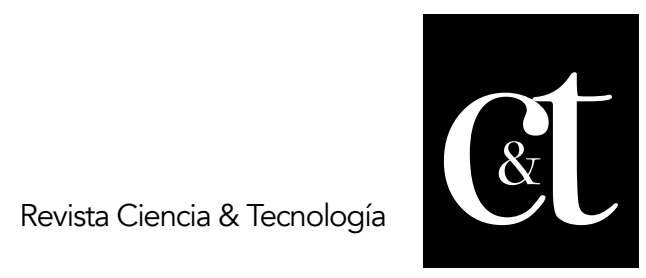

No. 25, 31 de enero de 2020

ISSN impreso: 1390 - 6321

ISSN online: 2661 - 6734

- El comercio electrónico y la competitividad exigen a las empresas adoptar una postura global que busque incrementar sus ingresos. Por ello la implementación herramientas de "almacenamiento en la nube" y Big Data se convierten en una estrategia necesaria que busque generar estos recursos de valor para la organización.

El pronóstico y control del pronóstico permitieron realizar la formulación del problema de investigación, que se presenta a continuación:

¿Cuáles son las ventajas y desventajas de la implementación del Big Data y el Cloud Computing en el proceso de toma de decisiones de las empresas que practican comercio electrónico?

A través de la metodología escogida se estableció el siguiente objetivo general:

- Analizar las ventajas y desventajas de la implementación del Big Data y Cloud Computing en el proceso de la toma de decisiones que realizan las empresas con comercio electrónico en el Ecuador.

Estableciendo los siguientes objetivos específicos:

- Determinar las ventajas y desventajas que obtienen las empresas con la utilización del Big Data en la minería de los datos no estructurados que provienen de la web.

- Proponer la implementación de la herramienta Cloud Computing como una estrategia de disminuir el costo de la adquisición de tecnología necesaria para el crecimiento competitivo de la organización.

\section{Justificación Teórica de la investigación}

El trabajo de investigación surgió de la necesidad de establecer el las ventajas y desventajas que tienen las herramientas tecnológicas como el Big Data y el Cloud Computing, las cuales "facilitan el conocimiento de las necesidades del cliente, creando valor y obteniendo competitividad indispensable para el desarrollo organizacional" (Aguirre \& Andrade, 2006).

Siguiendo la metodología seleccionada se presenta el esquema del método de las variables (gráfico 1) para establecer la influencia del social media en la competitividad de los negocios.

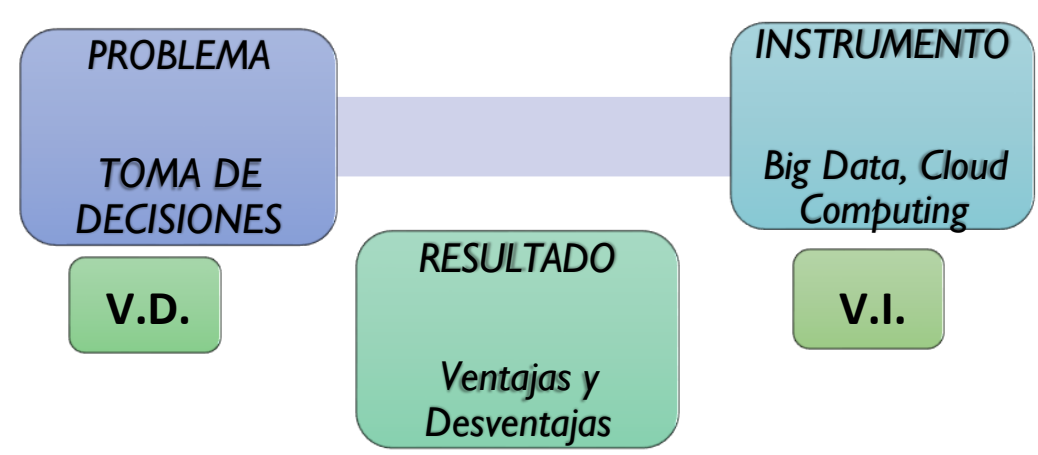

Gráfico 1. Método de las variables

Fuente: Elaboración propia 


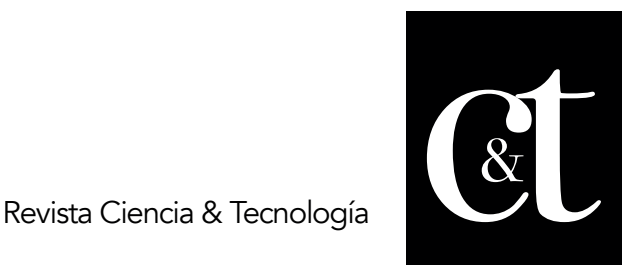

No. 25, 31 de enero de 2020

ISSN impreso: 1390 - 6321

ISSN online: 2661 - 6734

\section{Desarrollo}

Big Data y su creación de valor en los negocios

La transformación que se ha dado con la llegada de la Web 2.0 ha popularizado aspectos de la informática tales como Social Computing o "software social" y la nube informática o "Cloud Computing", actualmente el volumen de datos que se generan en nuestra Sociedad de la Información y el Conocimiento (SIC) son abundantes y con un crecimiento exponencial que se genera segundo a segundo. La denominación que predomina en la actualidad asociada a la problemática de "gestionar estas grandes cantidades de datos que no se pueden tratar con medios tradicionales" (Calero, 2013), es Big Data. El estudio de grandes cantidades de datos tomando como referencia estas propiedades permite la identificación rápida de patrones que ayudan a las empresas en el proceso de la toma de decisiones, a través de la minería de los datos, que se ha convertido en una herramienta muy valiosa de crecimiento empresarial en el mundo de los negocios.

Es necesario saber que los datos por sí mismos "aportan nada o incluso generar ruido, especialmente si se encuentran en volúmenes elevados, por lo que su procesado se hace indispensable" (Martínez \& Lara, 2014). Por ello la importancia de esta herramienta ya que permite a la empresa crear valor de los datos no estructurados, esto genera una información valiosa y necesaria para el administrador de empresas. Con la llegada de la web 2.0 y el protagonismo que adquiere el usuario, es decir han aumentado (Martínez \& Lara, 2014).

Algunas cifras permiten medir la magnitud de tal crecimiento. Según ComScore, "desde el 2007 al 2011 la audiencia mundial en sitios sociales registra un aumento del $174 \%$ y en junio del 2012 sumaban 1.258 millones de usuarios" (Martínez \& Lara, 2014). Los datos por plataformas también resultan espectaculares por su dimensión, tenemos por ejemplo que en marzo del 2014 "Facebook contaba con 802 millones de usuarios activos al día y, la comunidad de profesionales que se conectan a través de LinkedIn sumaba en abril de ese año, más de 300 millones de miembros" (Tascán, 2013).

Atendiendo al intercambio de contenidos destacan los 500 millones de tweets al día que registra Twitter, "las 100 horas de vídeo que cada minuto se suben a YouTube y los 25 mil millones de 'pins' que se contabilizan en Pinterest" (Martínez \& Lara, 2014).

La información y los mensajes que se intercambian en los Social Media, videos y audios que circulan en una dimensión semipública de la comunicación tienen perfiles de privacidad, por lo cual esta información no puede ser utilizada en su totalidad por las empresas, esto ha generado un debate sobre el respecto del derecho a la intimidad.

Herramientas del Big Data - Caso Hadoop y su creación de valor para la empresa La librería de software Apache Hadoop es un framework que permite el procesamiento distribuido de grandes conjuntos de datos a través de grupos de ordenadores que utilizan modelos de programación simples. La potencia real de Hadoop radica en que "puede manejar todos los tipos de datos de sistemas diferentes: estructurada, no estructurada, archivos log, imágenes, archivos de audio, los registros de comunicaciones, el correo electrónico casi cualquier cosa que se puede imaginar, con independencia de su formato nativo" (Lara, 2013). 


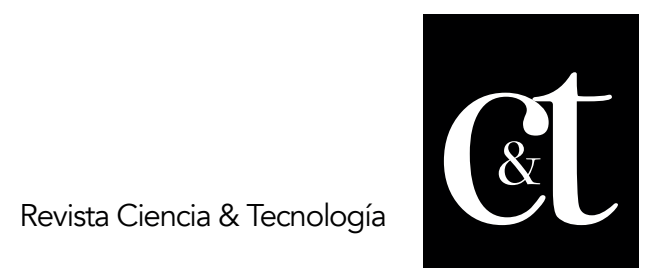

No. 25, 31 de enero de 2020

ISSN impreso: 1390 - 6321

ISSN online: 2661 - 6734

En el gráfico 1 se presenta el esquema de la herramienta Hadoop y el flujo de información que posee, adicional permite observar la depuración que se realiza para la obtención de valor, así también se muestra como suele "volcarse todo en el clúster Hadoop sin necesidad previa de un esquema" (Lara, 2013).

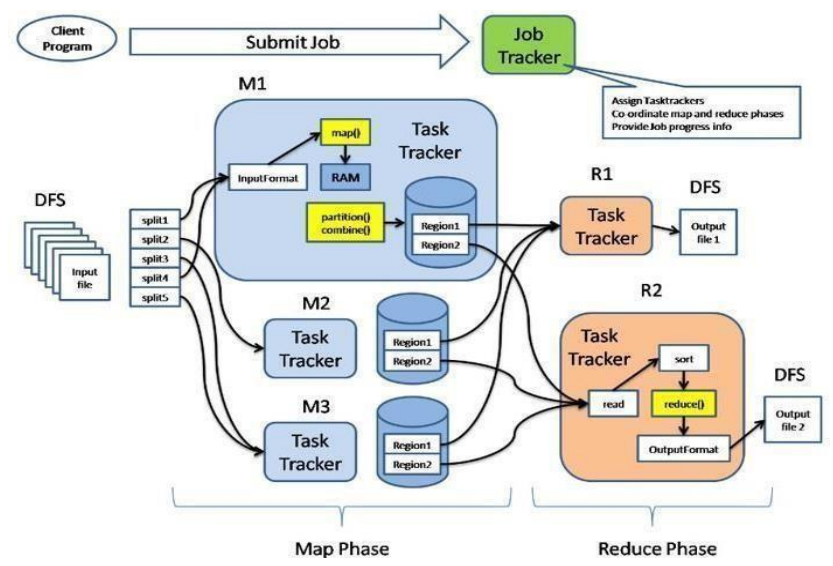

Gráfico 2. Esquema de Hadoop

Fuente: Lara (2013). Big Data \&Web Intelligence. Asunción: Repositorio Universidad Católica Nuestra Señora de la Asunción

Ejemplos de empresas que utilizan las herramientas Big Data

El Big Data es una tecnología que no sólo está en desarrollo, la misma es utilizada en la actualidad por empresas como eBay, Amazon, Facebook, Twitter, Netflix, IBM, The New York Times, LinkedIn, AOL, entre las más conocidas. Los campos de aplicación de Big Data son incalculables, por ahora se encuentran limitados exclusivamente a la generación de "nuevas ideas de las personas encargadas de desarrollar los proyectos o pensar en ideas de implementación de Big Data en un esquema particular" (Lara, 2013), entre los proyectos hechos que más utilizan Big Data se encontró:

Spotify: Es un servicio comercial de streaming de música que se inició en 2008 y desde entonces se ha registrado más de 24 millones de usuarios activos de los cuales 6 millones son usuarios de pago. Tiene millones de fans en Facebook. Cuenta con más de 20 millones de canciones en línea y cada día 20000 nuevas canciones se agregan a la base de datos. Los usuarios crearon más de 1 billón de listas de reproducción y más de $\$ 500$ millones de USD han sido pagados a los titulares de derechos desde el lanzamiento de Spotify.

Los usuarios de Spotify crean 600 Gigabytes de datos al día y 150 Gigabytes de datos por día a través de los diferentes servicios. Esto le permite la creación de información valiosa que obtiene de sus usuarios, un ejemplo de ello "fue en el 2013 donde Spotify utilizó los datos streaming para predecir los ganadores de los Premios Grammy de ese año, resultando 4 predicciones correctas de las 6 que realizó" (Lara, 2013).

Coca Cola: La empresa multinacional utiliza el Big Data para producir jugo de naranja con sabor consistente todo el año, aunque las naranjas utilizadas tienen una temporada alta de crecimiento de solo tres meses. Para ello han desarrollado un algoritmo llamado el "Black Book Model", que "combina varios conjuntos de datos, tales como imágenes satelitales, fecha de clima, las cosechas esperadas, las presiones de costos, preferencias de los consumidores regionales, información 


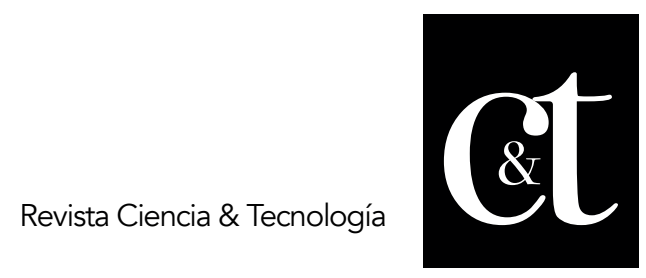

No. 25, 31 de enero de 2020

ISSN impreso: 1390 - 6321

ISSN online: 2661 - 6734

detallada acerca de la gran variedad de los 600 sabores diferentes que conforman una naranja" (Lara, 2013).

La arquitectura del algoritmo es una de las más complejas aplicaciones de análisis de negocios, porque combina hasta 1 trillón de variables de decisión para entregar consistentemente la mezcla óptima.

Netflix: Utiliza los hábitos de sus usuarios y clasificaciones de películas y los ejecuta a través de un algoritmo para generar el sistema de recomendación de 5 estrellas a medida de cada suscriptor.

A través del estudio de estas grandes corporaciones se puede evidenciar la gran utilidad que brinda el Big Data en el proceso de toma de las decisiones en las empresas, la información obtenida de los grandes volúmenes de datos ha generado valor para la organización, permitiendo el crecimiento de estas corporaciones y su permanencia en el mercado. Así también han creado nuevas herramientas para los negocios, convirtiendo al Big Data en una herramienta necesaria en la actualidad.

Siguiendo nuestra línea de investigación abordaremos las aplicaciones que se obtiene de la implementación del Cloud Computing.

\section{Capas del Cloud Computing}

El Cloud Computing se puede dividir en tres niveles (capas) en función de los servicios que actualmente están ofreciendo las empresas, en el futuro pueden surgir otras capas o clasificaciones.

- Infraestructura como servicio:

Se puede decir que es la parte física de la nube. En lugar de que los clientes tengan sus equipos en su propio lugar de trabajo, utilizan un alquiler de servicio y cancelan a un proveedor para que éste sea quien tenga todo ese equipamiento, ya sean discos duros o equipamiento de redes (Abelar \& Hernández, 2010) y, se encargue de toda la mantención y optimización de dicho equipamiento, el ejemplo de empresa que ofrece este tipo de servicio es Amazon Web Service con su servicio EC2 (Amazon Elastic Compute Cloud), es un "servicio web que proporciona tamaño variable de capacidad de cómputo en la nube y, está diseñado para hacer escala informática web más fácil para los desarrolladores" (Abelar \& Hernández, 2010).

\section{- Plataforma como servicio}

Se encuentra muy ligada a la capa "software como servicio, porque es la plataforma en donde se envuelve el software que pone a disposición el proveedor y es el medio de virtualización para el hardware que el cliente arrienda. La empresa que más servicios enfocados a esta capa es Google App Engine, "el cual permite desarrollar, compartir y alojar aplicaciones Web de terceros en su vasta infraestructura" (Abelar \& Hernández, 2010). 


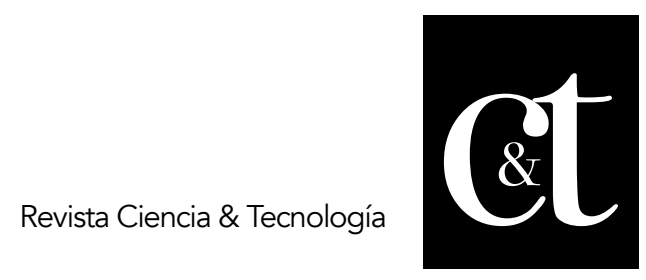

No. 25, 31 de enero de 2020

ISSN impreso: 1390 - 6321

ISSN online: 2661 - 6734

- Software como servicio

El proveedor de servicio pone a disposición de los clientes su propio software, creando así un ahorro para el cliente en la búsqueda de licencias, manteniendo actualizado siempre el sistema que necesita el cliente, un ejemplo de esta modalidad es Zetasoftware. En otro campo de la investigación se encontró que existen tres tipos de nubes que se utilizan en el mundo empresarial, entre ellas tenemos:

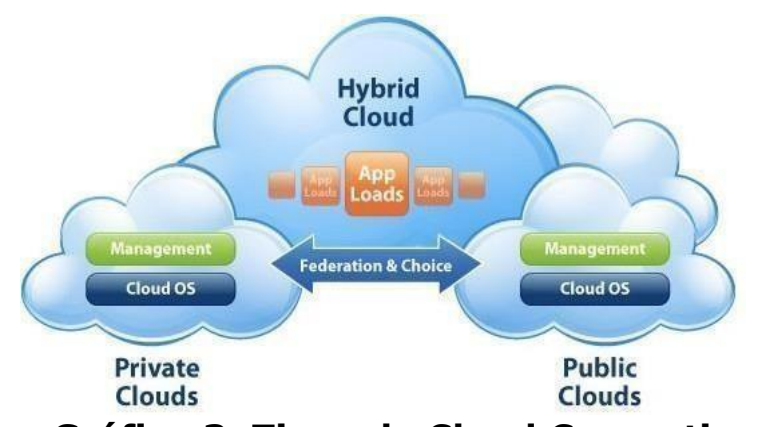

Gráfico 3. Tipos de Cloud Computing

Fuente y elaboración: Abelar, M., \& Hernández, I. (2010). Cloud Computing como solución de la gestión de las Pymes. Montevideo: Repositorio Universidad de la República

- Privadas

Las nubes privadas son manejadas por un solo cliente que controla qué aplicaciones debe correr y dónde, "son propietarios del servidor, red y disco y que pueden decidir qué usuarios están autorizados a utilizar la infraestructura" (Abelar \& Hernández, 2010).

- Públicas:

Las nubes públicas se manejan por terceras partes, y los trabajos de muchos clientes de diferentes pueden ser mezclados en los servidores, los sistemas de almacenamiento y otras infraestructuras de la nube. Es importante indicar que los usuarios finales no conocen qué trabajos de otros clientes pueden estar corriendo en el mismo servidor, red, discos como los suyos.

- Híbridas:

Las nubes híbridas son las más recomendadas para las empresas pequeñas, combinan los modelos de privada y pública, el propietario es dueño de una parte y comparte otras, actualmente las empresas perciben atracción por la nube híbrida por la facilidad de acoplamiento que tiene a las necesidades de los clientes.

Ventajas del Cloud Computing

Cloud Computing tiene muchas ventajas, entre las principales para el análisis de nuestro estudio se encontró: 


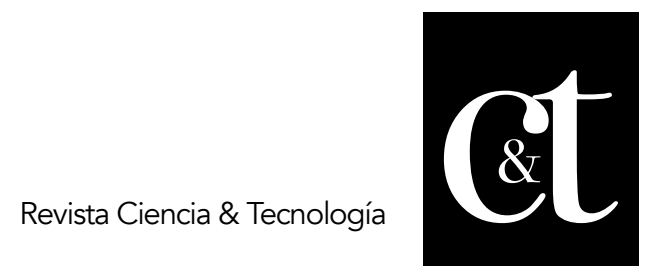

No. 25, 31 de enero de 2020

ISSN impreso: 1390 - 6321

ISSN online: 2661 - 6734

- Acceso: el usuario puede acceder a la información desde otra parte del mundo a cualquier hora.

- Costos Bajos: no existe la compra de licencia para el usuario, no existe compra y gasto en equipamiento de hardware y software, así también el usuario no tiene que preocuparse del mantenimiento ni actualización de sus equipos.

- Espacio de Almacenamiento. El cliente no utiliza espacio físico dentro de su empresa, y los archivos al encontrarse en la nube permitirán que el ordenador del cliente siempre se encuentre libre.

Desventajas de la Cloud Computing

Cloud Computing trae ventajas significativas, pero es muy importante saber que desventajas puede traer al manejarlo de manera incorrecta.

\section{- Dependencia}

En la utilización del Cloud Computing el usuario depende del proveedor, como lo indicó Steve Wozniak, cofundador de Apple ante esta creciente inquietud exclamó: "Cuanto más transfiramos todo a la web, en la nube, menos control vamos a tener de ello" (Mamani, 2014), es decir que si el proveedor por ejemplo se declararé en bancarrota y detuviese la provisión de servicios, el cliente podría experimentar serios inconvenientes en el acceso a sus datos y en consecuencia afectar la continuidad del negocio.

- Conexión a Internet

Una desventaja importante para el usuario podría ser qué "al no contar con una conexión a internet, no podría acceder a la nube, ni a sus datos" (Mamani, 2014); por ello como recomendación el cliente debe contar con un acceso a internet fiable y que la conexión no sea lenta.

\section{- Riesgo}

La seguridad y privacidad para el cliente es una preocupación constante, muchas empresas no están cómodas sabiendo que "sus datos están almacenados en un servidor virtual, del cual no se sabría con qué nivel de seguridad se cuenta" (Abelar \& Hernández, 2010)

\section{- Migración}

Si el usuario quiere cambiar su información a otro proveedor del servicio no será fácil transferir gran cantidad de datos de un proveedor a otro.

El Cloud Computing y su relación con el comercio electrónico

El Cloud Computing va creciendo y podemos verlo con el aumento de servicios y aplicaciones que se encuentran en la red, muchas entidades entendieron que usar una nueva forma para manejar la información como esta, ha generado ventajas y desventajas al incorporar esta tecnología en sus empresas. El término computación en la nube fue utilizado por el profesor Kenneth K. Chellapa, quien los describió en 1997 en la conferencia de Informa en Dallas como un "paradigma de computación donde los límites de la computación serán determinados por razones económicas más que las limitaciones técnicas" (Abelar \& Hernández, 2010); otra definición es la que ofreció la firma de Consultoría de Accenture es: "el aprovisionamiento dinámico de las capacidades de TI (hardware, software o servicios) de terceros a través de una red" (Alvarez \& Díaz, 2011). 


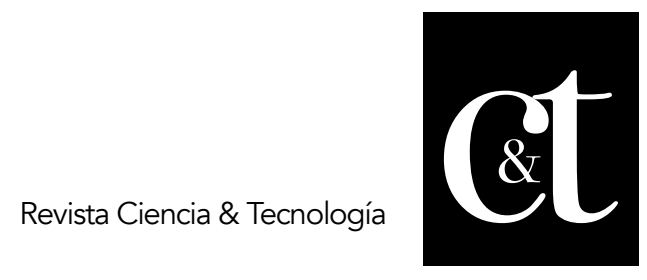

No. 25, 31 de enero de 2020

ISSN impreso: 1390 - 6321

ISSN online: 2661 - 6734

A través de la investigación se puede citar uno de los conceptos que estableció la IEE Computer Society, al indicar que la "computación en la nube" es "una tendencia en Tecnologías de Información que mueve los datos y el procesamiento fuera del escritorio y las computadoras portátiles hacía grandes centros de datos" (Abelar \& Hernández, 2010), es decir que detrás del surgimiento del Cloud Computing incluye la sobre capacidad de los grandes centros de datos corporativos de la actualidad. El surgimiento del Cloud Computing ha evidenciado la eficiente entrega de demanda de software, hardware y datos como servicio, logrando que los usuarios satisfagan sus necesidades a un menor costo debido a que pagarán por lo que realmente utilicen del servicio (Abelar \& Hernández, 2010). En la actualidad esta tecnología permite a los empresarios adoptar estrategias que sólo se fundamentan en el negocio que realizan, porque el Cloud Computing permite el ahorro "en la parte financiera de la implementación de la maquinaria que necesita la empresa, el ahorro en costos de mantenimiento y de personal especializado encargado de cuidar la información de la empresa" (Álvarez \& Díaz, 2011).

\section{Conclusiones}

Para concluir nuestra investigación es importante mencionar que es incuestionable la creciente cantidad de información que se genera segundo a segundo en nuestro planeta, la cual puede ser estructurada, semiestructurada o no estructurada, así también se estableció la importancia de la implementación del Big Data y el Cloud Computing son excelentes herramientas para el crecimiento de las empresas.

La información que se obtuvo a través de la herramienta Big Data es considerada de enorme valor a cualquier empresa, organización "o institución que la requiere (la mayoría de las veces en tiempo real), representa este tipo de información un requisito innegable para la supervivencia de muchas organizaciones y empresas" (Jiménez, 2014).

La creación de conocimiento demanda la búsqueda de talento humano capacitado en analizar estos cubos de información, los llamados: "científicos de datos" están creciendo en el mercado laboral muy rápidamente, "por lo que se requiere una continua preparación en el conocimiento de todas las posibilidades que se presentan en la utilización de estas herramientas tecnológicas" (Jiménez, 2014). A través de la presente investigación se estableció las siguientes conclusiones sobre la herramienta Big Data en los negocios:

En el año 2010 el término Big Data era desconocido, a mediados del 2011 se convertía en una palabra que aparecería con frecuencia entre las últimas tendencias. Este artículo ha presentado un análisis de las ventajas y desventajas de la implementación del Cloud Computing y la participación activa en la inteligencia de los negocios del Big Data, así podemos determinar que la "analítica del Big Data se está desarrollando gracias a la participación de la Ingeniería Estadística "en coordinación con las técnicas de Inteligencia Artificial, por esto es importante afirmar que Big Data representa un nuevo paradigma dentro del análisis dedatos" (Jiménez, 2014).

Big Data es una herramienta que ha ganado gran notoriedad los últimos años y se relaciona estrechamente con la Web Intelligence, se puede indicar entonces que ambas en conjunto se convierten en una estrategia interesante a la hora de desarrollar aplicaciones web para los consumidores, sitios como Amazon, Google, 


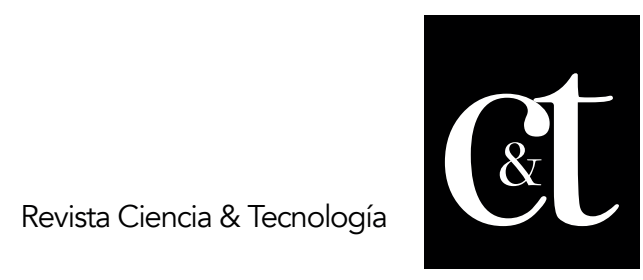

No. 25, 31 de enero de 2020

ISSN impreso: 1390 - 6321

ISSN online: 2661 - 6734

siendo una tecnología relativamente nueva la utilizan cotidianamente (Abelar \& Hernández, 2010).

\section{Referencias bibliográficas}

Abelar, M., \& Hernández, I. (2010). Cloud Computing como solución de la gestión de las Pymes. Montevideo: Repositorio Universidad de la República.

Aguirre, G., \& Andrade, H. (2006). BI Business Intelligence. Guayaquil: Espol. Bonales, J., \& Zamora, A. (2015). Variables e índices de Competitividad de las empresas exportadoras, utilizando el PLS. CIMEXUS Vol. X, No. 2, 20.

Calero, A. (2013). Nueva Ciencia y tecnología de Redes. Una nueva forma de tratar la complejidad. TELOS 95, 4.

Crespo, P. (2013). El uso de las TICS como herramienta para la internacionalización de las PYMES en el Ecuador. Cuenca: Repositorio Universidad del Azuay.

Cueva, C. (2014). Determinación de la línea de base para la aplicación de sistemas BI en el Ecuador. Quito: Repositorio Universidad de las Fuerzas Armadas.

El Comercio. (19 de Julio de 2015). El 82\% de pymes de Ecuador accede a Internet, pero su uso se limita a enviar correos y tareas administrativas.

El Universo. (1 de junio de 2016). Ecuador es el segundo país que más emprende en el mundo, pero los negocios no se consolidan, p. 1.

Jiménez, C. (2014). Big Data. Un nuevo paradigma de análisis de datos. Anales de mecánica y electricidad. nov-dic., pp.10-16.

Lara, J. (2013). Big Data \& Web Intelligence. Asunción: Repositorio Universidad Católica Nuestra Señora de la Asunción.

Mamani, J. (2014). Ventajas y Desventajas de Cloud Computing. Revista de inforinformación, tecnología y sociedad, 3.

Martínez, S., \& Lara, P. (2014). El Big Data transforma la interpretación de los medios sociales. El profesional de la información. vol.23, №. 6.

Tascán, M. (2013). Big Data: Pasado, presente y futuro. Telos 95, 4. 